\title{
Visualization of Mitochondria and Some Other Cytoplasmic Structures in Hematoxylin-eosin Stained Sections by Fluorescence Microscopy
}

\author{
By \\ Kazuo YAMASHITA, Takashi WAKABAYASHI and Kayo ADACHI \\ Department of Anatomy, Nagoya University School of Medicine, \\ Tsurumai-cho, Showa-ku, Nagoya 466, Japan \\ -Received for Publication, August 25, 1986-
}

\begin{abstract}
Key Words: Hematoxylin and eosin stain, Fluorescence microscopy, Mitochondria, Juxtaglomerular granules, Lipofuscin granules

Summary: In hematoxylin (H)-eosin (E) stained, paraffin sections prepared after fixation in $10 \%$ neutral buffered formalin, fluorescence microscopy demonstrated mitochondria, juxtaglomerular granules and some other structures in various tissues. Numerous fluorescent mitochondria were observable in hepatocytes under violet or green excitation. In hydrazine-fed mice, mitochondria in hepatocytes were large. In pancreatic acinar cells, bright filamentous mitochondria were demonstrated in the basal cytoplasm and intensely fluorescent secretory granules were in the luminal cytoplasm. Parietal cells of the stomach showed densely packed, fluorescent granular mitochondria. In epithelial cells of the kidney, rod-shaped fluorescent mitchondria exhibited a distinct striation in the basal cytoplasm. Juxtaglomerular cells in the afferent arteriole contained secretory granules which were more intensely fluorescent than mitochondria. Lipofuscin granules were autofluorescent in H-E stained sections of the ovarian interstitial cells, testicular Leydig cells and adrenal cortical cells.
\end{abstract}

The aim of the present study is to show that mitochondria, juxtaglomerular granules and some other cytoplasmic structures can clearly be recognized in routine hematoxylineosin (H-E) stained, paraffin sections in a fluorescence microscope. These findings indicate that much useful information, not available in $\mathrm{H}-\mathrm{E}$ stained sections by ordinary light microscopy, can be obtained from the same sections by fluorescence microscopy without any additional stains. This double microscopy may be of great value in the fields of experimental and diagnostic morphology. To our knowledge, such attempts have not been performed.

\section{Materials and Methods}

Most of the present observations were carried out on adult mouse tissues. The animals were anesthetized and perfused through the ascending aorta with $10 \%$ buffered formalin. The liver, pancreas, kidney, salivery gland, stomach, adrenal gland, ovary, and testis were then removed, immersed overnight in the perfusate, dehydrated and embedded in paraffin. Mitochondria pellet was prepared from rat liver with a routine method and processed similarly through paraffin or smeared on a glass slide for light microscopy. A part of the pellet was served for electron microscopy after postosmication and Epon-embedding. For some illustrative experiments livers 
of normal and hydrazine-fed young mice ( $0.1 \%$ hydrazine for five days), adult mice and house musk shrews (Suncus murinus) were fixed with Zamboni's solution by perfusion and processed through paraffin or for electron microscopy.

Paraffin sections were cut at 2 to $4 \mu \mathrm{m}$ stained with $\mathrm{H}$ and $\mathrm{E}$. The $\mathrm{H}$ solution was prepared according to a modified Carrazi formula $^{8)}$ as follows:

$\begin{array}{lc}\text { Hematoxylin } & 3 \mathrm{~g} \\ \text { Distilled water } & 800 \mathrm{ml} \\ \text { Potassium alum } & 50 \mathrm{~g} \\ \text { Sodium iodate } & 0.6 \mathrm{~g} \\ \text { Glycerol } & 200 \mathrm{ml}\end{array}$

Hematoxylin was dissolved in the water and other gradients were added using gentle heat if necessary. The staining time was 4 min.

The $\mathrm{H}$ stained sections were counterstained for $2 \mathrm{~min}$ in the following dichromatic eosin solution ${ }^{4)}$.

$1 \%$ aqueous solution of eosin $\mathrm{Y} 100 \mathrm{ml}$ (C.I. 45380)

$1 \%$ aqueous solution of phloxine $\mathrm{B} 10 \mathrm{ml}$ (C.I. 45410)

95\% ethanol $780 \mathrm{ml}$

Glacial acetic acid $4 \mathrm{ml}$

Sections were observed with a Zeiss universal microscope equipped with an ordinary transillumination system and an epifuluorescence excitation system (100 W $\mathrm{Hg}$ ). The filter sets (exciter filter, dichromatic beam splitter, barrier filter) used were Ultraviolet (UV excitation filter), Violet (V), Blue (B) and Green (G).

\section{Results}

Liver cells: Figure 1 is an ordinary light micrograph of a $\mathrm{H}-\mathrm{E}$ stained section of the periportal region of the mouse liver. Figure 2 is the same portion seen in Figure 1, where fluorescent granules are well demonstrated in hepatocytes. The granules show distinct zonal difference in size. In the mouse liver, the size decreases toward the centrilobular region (Figs. 2 and 3). In the house musk shrew liver, however, the granules are small in the periportal region while large in the centrilobular region (Figs. 4 and 5). $\mathrm{H}-\mathrm{E}$ stained paraffin sections of rat liver mitochondria pellet, which has been confirmed to consist of mitochondria by electron microscopy, show only packed, bright granules under epifluorescence illumination. Stained smears from the pellet also reveal single fluorescent granules (Fig. 6). As shown in Fig. 7, electron microscopy demonstrates marked enlargement of mitochondria in hepatocytes of mice fed $1 \%$ hydrazine for 5 days. The corresponding epifluorescence photomicrograph shows a picture of enlarged fluorescent granules (Fig. 8).

Pancreatic acinar cells: When $\mathrm{H}$ and $\mathrm{E}$ stained sections of the mouse pancreas are observed under epifluorescence excitation, secretory granules of acinar cells and secreted material in the ductule give a strong fluorescence (Fig. 9). In the basal cytoplasm of acinar cells, conspicuous fluorescent filaments appear on a black background (Fig. 9). The filaments appear to correspond to mitochondria in morphology and distribution.

Kidney: Epithelial cells of distal tubules of the mouse kidney have numerous fluorescent long rods in the basal cytoplasm (Fig. 10). The rods, oriented parallel to the long cell axis, appear as a prominent basal striation. In addition, intensely fluorescent granules are seen in juxtaglomerular cells located in the afferent arteriole just proximal to the entrance into the glomerulus. The granules are not seen under transillumination. Even under epifluorescence illumination, they are not observed in sections strained with $\mathrm{H}$ alone. In sections stained only with the dichromatic eosin solution, the fluorescence is very weak. When stained with $\mathrm{H}$ and $\mathrm{E}$, the granules appear as intensely red with excitation filter $G$ and bright 
white-yellow with filter V (Figs. 11 and 12). Particularly, under violet excitation the granules are well discernible from mitochondria in color and density.

Salivary gland: Columnar epithelium of the striated segments in the gland exhibits a parallel fluorescent striation in the basal cytoplasm.

Gastric gland: In cytoplasm of parietal cells, densely packed, bright granules are seen under epifluorescence illumination (Fig. 13). Zymogenic cells have secretory granules faintly discernible by fluorescence microscopy. The basal cytoplasm, however, contains bright fine filamentous mitochondria which are similar to those in the pancreatic acinar cells.

The fluorescence of the filaments or granules seen in the cells described above is the strongest under green excitation and next under violet excitation, but is faint by filter B or UV. The fluorescence appears red under green excitation and bright orange under violet excitation. The fluorescence occurs in sections stained only with either eosin or phloxine, but not in sections with hematoxylin alone.

Others: By fluorescence microscopy, irregularly shaped, large bright structures are seen in various cells such as cells of the zona reticularis of the adrenal gland, interstitial gland cells of the ovary and Leydig cells of the testis. The structures are observed after either $\mathrm{H}$ or $\mathrm{E}$ staining under violet excitation. In H-E stained sections, the violet or blue excitation differentiates well the structure from the others on the basis of their color. Red blood cells and collagenous and elastic fibers exhibit fluorescence. Collagenous fibers show strong fluorescence under violet excitation.

\section{Discussion}

The H-E staining in combination with fluorescence microscopy has been shown to provide a practical, readily applicable microscopic approach to demonstrate and identify certain cytoplasmic structures which are difficult to be visualized by ordinary light microscopy on the same section.

As generally known, mitochondria can not be differentiated in ordinary histological sections. The present fluorescence microscopy on H-E stained sections, however, has shown that in various cells such as in the liver, pancreas, kidney and salivery gland, mitochondria may appear as fluorescent granules or filaments. The fluorescence microscopy of H-E stained paraffin sections of mitochondrial pellet from rat liver has shown that the pellet consists almost entirely of rounded fluorescent granules. In stained smears prepared from the pellet, single fluorescent granules have clearly been seen. Thus, it is most likely that the fluorescent granules in question are mitochondria. Two additional illustrative studies has been carried out on the zonal difference of mitochondria in the hepatic lobule and morphological changes of mitochondria in hepatocytes of hydrazine-fed mice. The quantitative electron microscopy demonstrated that mitochondria are larger in the periportal region than in the centrilobular area in the rat and mouse $^{3)}$. The situation is reverse in the house musk shrew (Yamashita and Kitoh, unpublished data). Mitochondria are greatly enlarged in hydrazine-fed mice ${ }^{7)}$. These previous findings have been confirmed by the present fluorescence microscopy. This means not only that the conclusion on the identification of mitochondria in H-E stained sections is further confirmed, but also that the present method is successfully applicable to experimental or diagnostic approaches.

The juxtaglomerular cells has not been 
easily recognized with routine stains. Various techniques have been published for the demonstration ${ }^{1-2}(, 5-6)$. The present fluorescent microscopy can distinctly reveal the specific granules in the juxtaglomerular cells. Lipofuscin granules are known to exhibit an autofluorescence. The granules that are observed clearly under violet excitation in $\mathrm{H}-\mathrm{E}$ stained sections of the ovarian interstitial cells, adrenal cortical cells and testicular Leydig cells are thought to be lipofuscin granules.

The fluorescence in $\mathrm{H}-\mathrm{E}$ stained sections is mainly due to the fluorescein dyes used, $\mathrm{E}$ and phloxine(P), because fluorescent structures other than juxtaglomerular and lipofuscin granules are generally eosinophilic in ordinary light microscopy. Hematoxylin may play a role in the elimination of some fluorescence since non or weakly fluorescent structures stained dark blue with $\mathrm{H}$. For example, nuclei fluoresced weakly in sections stained with $\mathrm{E}$ or $\mathrm{P}$ alone, but not after $H$ stain. Thus, the fluorescence induced by $\mathrm{E}$ and $\mathrm{P}$ becomes clearly evident against the black background. However, we have observed that $\mathrm{H}$ is involved in the increase in fluorescence in the case of juxtaglomerular granules. The nature of the fluorescent complex formed under this condition is unclear.

This work shows that fluorescence microscopy of the routine stained sections expands our information gained. The concurrent transmitted light and epifluorescence microscopy will find many applications in experimental and diagnostic histology.

\section{Acknowledgements}

We wish to thank Mr. S. Sasaki and Mr, S. Terada for their technical assistance. We are also very grateful to Dr. T. Ito (Emeritus Professor of Hokkaido University) for his critical reading of the manuscript.

\section{References}

1) Edelman, R. and Hartroft, P.M.. Localization of renin in juxtaglomerular cells of rabbit and $\operatorname{dog}$ through the use of fluorescence antibody technique. Clin. Res., 9: 1069-1077, 1961.

2) Harada, K.: Rapid demonstration of juxtaglomerular granules with alcoholic crystal violet. Stain Technol., 45: 71-74, 1970.

3) Loud, A.V.: Quantitative stereological description of the ultrastructure of normal rat liver parenchymal cells. J. Cell Biol., 37: $27-46,1968$.

4) Sheehan, D.C., and Hrapchak, B.B.: Nuclear and cytoplasmic stains (Chapter 8). Theory and Practice of Histotechnology, Second edition, pp. 137-18, Mosby, St. Louis, 1980.

5) Smith, C.L.: Rapid demonstration of juxtaglomerular granules in mammals and birds. Stain Technol., 41: 291-294, 1966.

6) Tanaka, T., Gresik, E.W., Michelakis, A.M. and Barka, T.. Immunocy tochemical localization of renin in kidneys and submandibular glands of $S W R / J$ and $C 57 \mathrm{BL} / 6 \mathrm{~J}$ mice. J. Histochem. Cy tochem., 28: 1113-1118, 1980.

7) Wakabayashi, T., Horiuchi, M., Sakaguchi, M., Onda, H. and Misawa, K.: Induction of megamitochondria in the mouse and rat livers by hydrazine. Exp. Mol. Pathol., 39: 139-153, 1983.

8) Watanabe, T.: Detailed exposition on routine standing methods (Chapter 2). Staining Methods (Part 2). Editor Jap. Soc. Pathol. Manuals of Pathological Techniques (Vol. 3). pp. 35-44. (in Japanese). Ishiyaku Shuppan, Tokyo, 1985. 


\section{PLATES}




\section{Explanation of Figures}

\section{Plate I}

Fig. 1. Transmitted light photomicrograph of a paraffin section of a periportal region of the hepatic lobule from a normal mouse. Hematoxylin and eosin (H-E). $\times 750$.

Fig. 2. Epifluorescence photomicrograph of the same field as in Fig. 1. Distinct fluorescent granules appear in the hepatocytes. Violet excitation, $\times 750$.

Fig. 3. Epifluorescence phtomicrograph of a H-E stained section of a centrilobular region from the same mouse as in Fig. 2. Note distinct differences of the size of hepatocytes and that of bright cytoplasmic granules between the two regions. Violet excitation, $\times 750$.

Fig. 4. Epifluorescence photomicrograph of a H-E stained section of periportal hepatocytes of a normal house musk shrew (Suncus murinus). Green excitation, X 1,570.

Fig. 5. Epifluorescence photomicrograph of a H-E stained section of centrilobular hepatocytes of the same animal as in Fig. 7. Compare the cell size and the size of cytoplasmic granules. Two large bright figures are red blood cells. Green excitation, $\times 1,570$.

Fig. 6. Epifluorescence photomicrograph of a H-E stained smear obtained from a rat mitochondrial suspension. Bright round particles are seen. Green excitation, X 1,570. 
Plate I
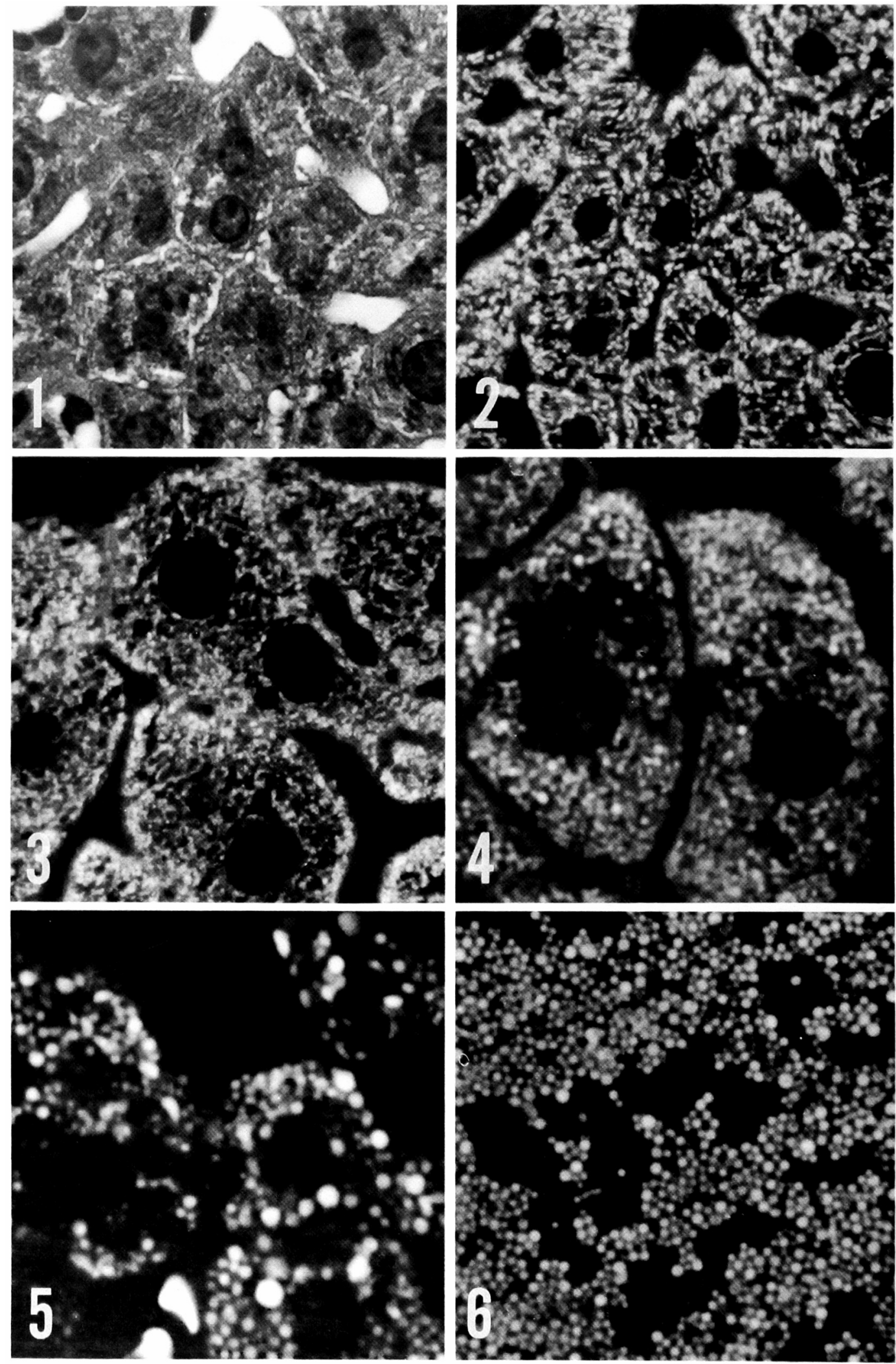


\section{Plate II}

Rig. 7. Eleetron micrograph of altered hepatocytes of a periportal region from a young mouse fed $1 \%$ hydrazine for five days. Note remarkably enlarged mitochondria. $\times 5,140$.

Inset: higher magnification of megamitochondria. $\times 19,550$.

Fig. 8. Epifluorescene photomicrograph of a H-E stained section of a periportal region of the same mouse as in Fig. 7. The hepatocy tes show large bright granules. Violet excitation, $X 630$.

Fig. 9. Epifluorescence photomicrograph of a H-E stained section of a portion of the mouse pancreas. Secretory granules of the acinar cells fluoresce intensely. In the basal cytoplasm, fluorescent filaments are clearly seen. Green excitation, X 1,250. 

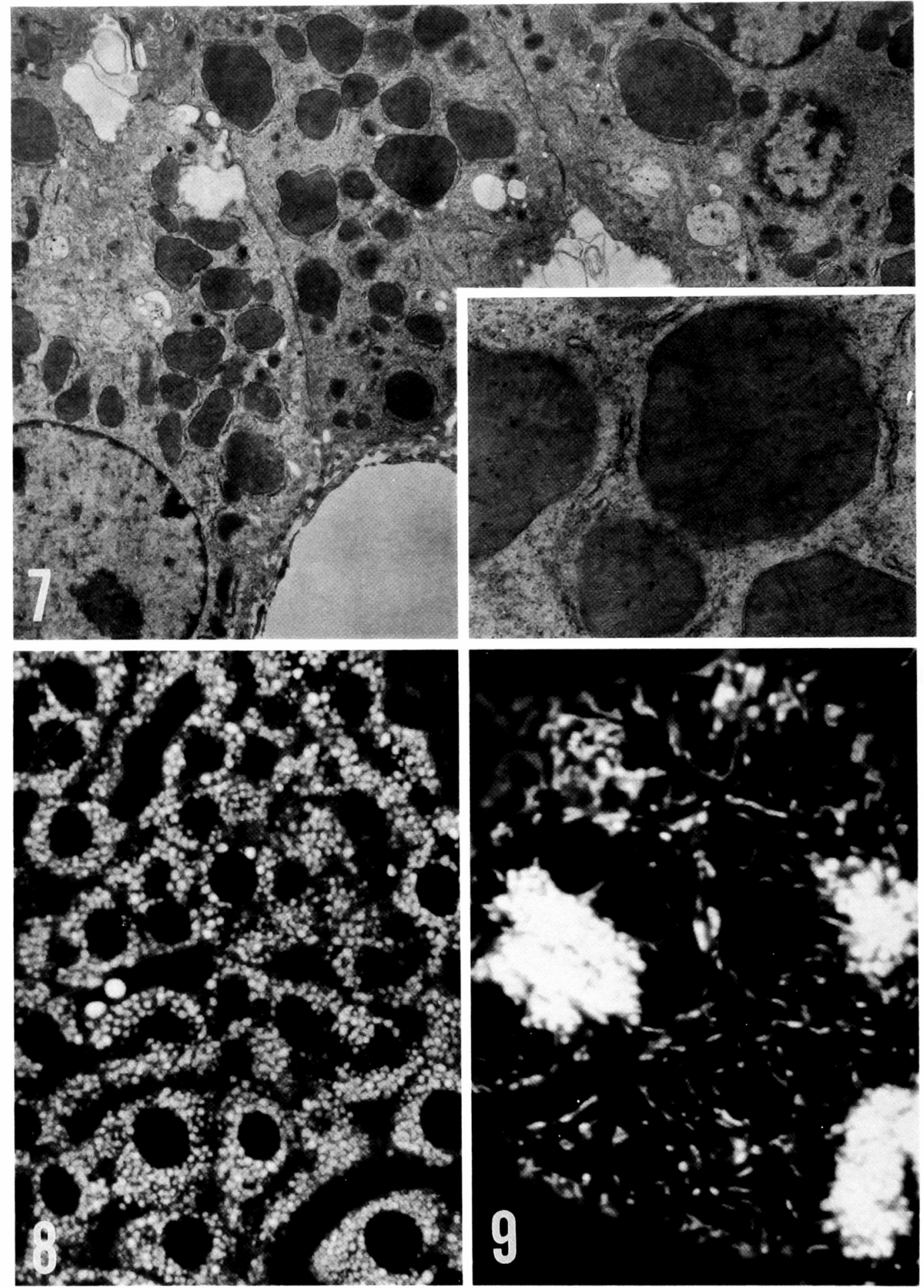


\section{Plate III}

Fig. 10. Epifluorescence photomicrograph of a H-E stained section of the mouse kidney. In the basal cytoplasm of distal tubule cells a marked fluorescent striation is observed. Large proximal tubule cells contain bright rod-shaped structures. Violet Excitation, $\times 630$.

Fig. 11. Epifluorescence photomicrograph of a H-E stained section of the mouse kidney. The juxtaglomerular cells contain highly fluorescent granules. Violet excitation, $\times 500$.

Fig. 12. High magnification epifluorescence photomicrograph of mouse juxtaglomerular cells. Large, intensely fluorescent granules are seen. Violet excitation, $X 1,250$.

Fig. 13 Epifluorescence photomicrograph of a H-E stained section of the mouse stomach. Parietal cells are densely packed with bright round bodies, among which negative images possibly of secretory canaliculi are recognized. Chief cells show pale granules in the luminal cytoplasm and fine bright filaments in the basal portion. Green Excitation, $\times 630$. 

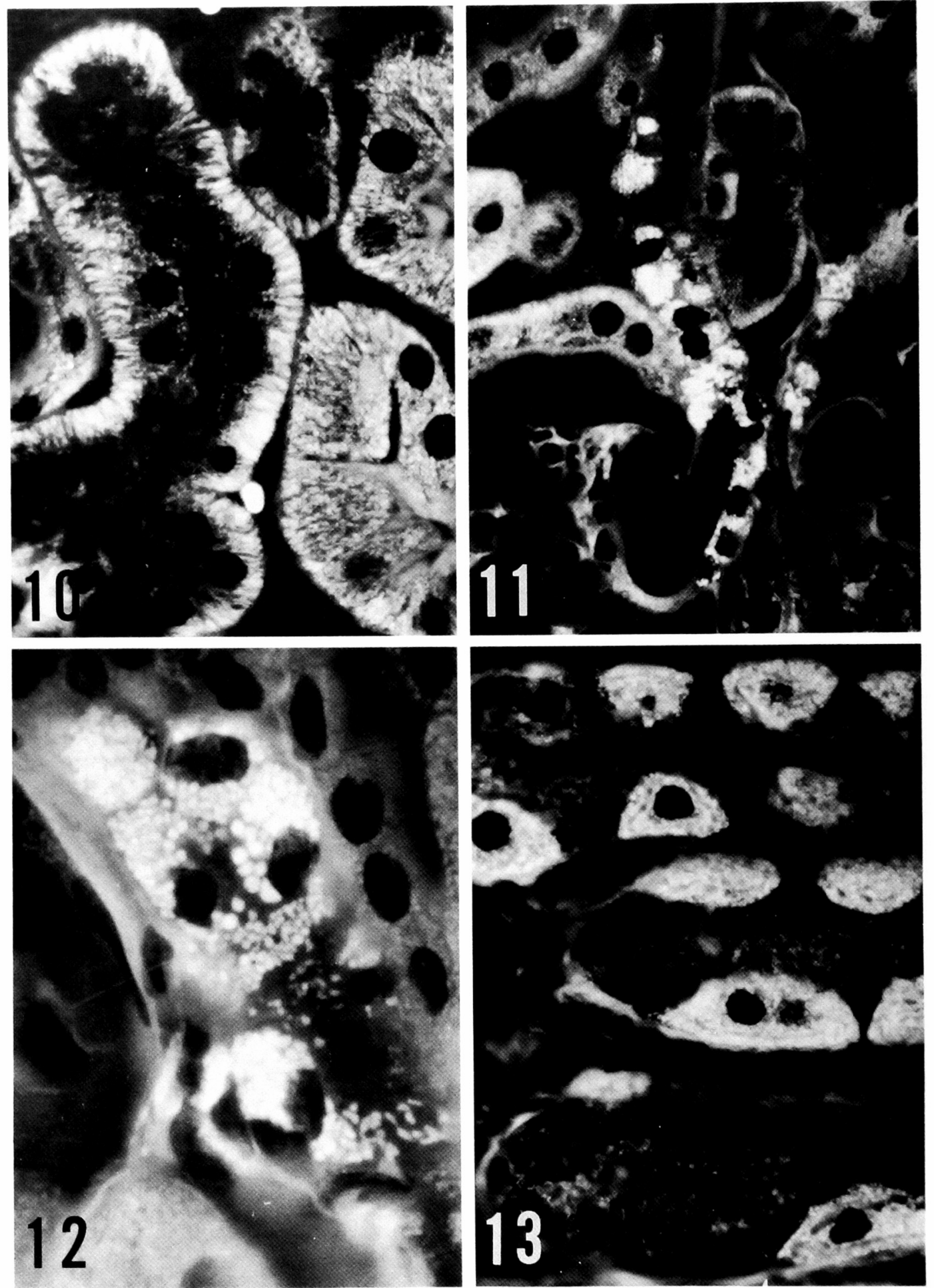